Supporting Information for

\title{
Intermolecular Covalent $\pi-\pi$ Bonding Interaction Indicated by Bond Distances, Energy Bands, and Magnetism in Biphenalenyl Biradicaloid Molecular Crystal
}

\author{
Jingsong Huang* and Miklos Kertesz* \\ Department of Chemistry, Georgetown University \\ $37^{\text {th }} \&$ O Street, Washington, D.C. 20057-1227 \\ E-mail: huangj2@georgetown.edu; kertesz@georgetown.edu
}

Complete author list of ref 26:

Frisch, M. J.; Trucks, G. W.; Schlegel, H. B.; Scuseria, G. E.; Robb, M. A.; Cheeseman, J. R.; Montgomery, Jr., J. A.; Vreven, T.; Kudin, K. N.; Burant, J. C.; Millam, J. M.; Iyengar, S. S.; Tomasi, J.; Barone, V.; Mennucci, B.; Cossi, M.; Scalmani, G.; Rega, N.;

Petersson, G. A.; Nakatsuji, H.; Hada, M.; Ehara, M.; Toyota, K.; Fukuda, R.; Hasegawa, J.; Ishida, M.; Nakajima, T.; Honda, Y.; Kitao, O.; Nakai, H.; Klene, M.; Li, X.; Knox, J. E.; Hratchian, H. P.; Cross, J. B.; Adamo, C.; Jaramillo, J.; Gomperts, R.; Stratmann, R. E.; Yazyev, O.; Austin, A. J.; Cammi, R.; Pomelli, C.; Ochterski, J. W.; Ayala, P. Y.; Morokuma, K.; Voth, G. A.; Salvador, P.; Dannenberg, J. J.; Zakrzewski, V. G.; Dapprich, S.; Daniels, A. D.; Strain, M. C.; Farkas, O.; Malick, D. K.; Rabuck, A. D.; Raghavachari, K.; Foresman, J. B.; Ortiz, J. V.; Cui, Q.; Baboul, A. G.; Clifford, S.; Cioslowski, J.; Stefanov, B. B.; Liu, G.; Liashenko, A.; Piskorz, P.; Komaromi, I.; Martin, R. L.; Fox, D. J.; Keith, T.; Al-Laham, M. A.; Peng, C. Y.; Nanayakkara, A.; Challacombe, M.; Gill, P. M. W.; Johnson, B.; Chen, W.; Wong, M. W.; Gonzalez, C.; Pople, J. A. 


\begin{tabular}{|c|c|c|c|c|c|c|c|}
\hline & $x$ & & 7 & & $x$ & 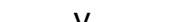 & 7 \\
\hline$C$ & 7.539954 & 0.000000 & 0.000000 & $\mathrm{H}$ & 5.159464 & 3.402001 & 0.000000 \\
\hline C & 6.858344 & 1.220918 & 0.000000 & $\mathrm{H}$ & 5.159464 & -3.402001 & 0.000000 \\
\hline C & 5.449790 & 1.251302 & 0.000000 & $\mathrm{H}$ & -5.159464 & 3.402001 & 0.000000 \\
\hline C & 4.648426 & 2.441718 & 0.000000 & $\mathrm{H}$ & -5.159464 & -3.402001 & 0.000000 \\
\hline C & 3.262688 & 2.418414 & 0.000000 & $\mathrm{H}$ & 8.626744 & 0.000000 & 0.000000 \\
\hline C & 2.581210 & 1.174658 & 0.000000 & $\mathrm{H}$ & 7.418028 & 2.153472 & 0.000000 \\
\hline C & 3.374107 & 0.000000 & 0.000000 & $\mathrm{H}$ & 7.418028 & -2.153472 & 0.000000 \\
\hline C & 2.581210 & -1.174658 & 0.000000 & $\mathrm{H}$ & 2.715408 & 3.354529 & 0.000000 \\
\hline C & 3.262688 & -2.418414 & 0.000000 & $\mathrm{H}$ & 2.715408 & -3.354529 & 0.000000 \\
\hline C & 4.648426 & -2.441718 & 0.000000 & $\mathrm{H}$ & -2.715408 & 3.354529 & 0.000000 \\
\hline C & 5.449790 & -1.251302 & 0.000000 & $\mathrm{H}$ & -7.418028 & 2.153472 & 0.000000 \\
\hline C & 6.858344 & -1.220918 & 0.000000 & $\mathrm{H}$ & -8.626744 & 0.000000 & 0.000000 \\
\hline C & 4.768851 & 0.000000 & 0.000000 & $\mathrm{H}$ & -7.418028 & -2.153472 & 0.000000 \\
\hline C & 1.202831 & 0.726912 & 0.000000 & $\mathrm{H}$ & -2.715408 & -3.354529 & 0.000000 \\
\hline C & 1.202831 & -0.726912 & 0.000000 & $\mathrm{H}$ & 0.000000 & 3.113164 & 2.147635 \\
\hline C & 0.000000 & -1.451481 & 0.000000 & $\mathrm{H}$ & 0.000000 & 5.593402 & 2.151382 \\
\hline C & -1.202831 & -0.726912 & 0.000000 & $\mathrm{H}$ & 0.000000 & 6.842826 & 0.000000 \\
\hline C & -1.202831 & 0.726912 & 0.000000 & $\mathrm{H}$ & 0.000000 & 5.593402 & -2.151382 \\
\hline C & 0.000000 & 1.451481 & 0.000000 & $\mathrm{H}$ & 0.000000 & 3.113164 & -2.147635 \\
\hline C & -3.374107 & 0.000000 & 0.000000 & $\mathrm{H}$ & 0.000000 & -3.113164 & 2.147635 \\
\hline C & -2.581210 & 1.174658 & 0.000000 & $\mathrm{H}$ & 0.000000 & -5.593402 & 2.151382 \\
\hline C & -3.262688 & 2.418414 & 0.000000 & $\mathrm{H}$ & 0.000000 & -6.842826 & 0.000000 \\
\hline C & -4.648426 & 2.441718 & 0.000000 & $\mathrm{H}$ & 0.000000 & -5.593402 & -2.151382 \\
\hline C & -5.449790 & 1.251302 & 0.000000 & $\mathrm{H}$ & 0.000000 & -3.113164 & -2.147635 \\
\hline C & -6.858344 & 1.220918 & 0.000000 & & & & \\
\hline C & -7.539954 & 0.000000 & 0.000000 & & & & \\
\hline C & -6.858344 & -1.220918 & 0.000000 & & & & \\
\hline C & -5.449790 & -1.251302 & 0.000000 & & & & \\
\hline C & -4.648426 & -2.441718 & 0.000000 & & & & \\
\hline C & -3.262688 & -2.418414 & 0.000000 & & & & \\
\hline C & -2.581210 & -1.174658 & 0.000000 & & & & \\
\hline C & -4.768851 & 0.000000 & 0.000000 & & & & \\
\hline C & 0.000000 & 2.945930 & 0.000000 & & & & \\
\hline C & 0.000000 & -2.945930 & 0.000000 & & & & \\
\hline C & 0.000000 & 3.658518 & 1.207873 & & & & \\
\hline C & 0.000000 & 5.054470 & 1.207458 & & & & \\
\hline C & 0.000000 & 5.755998 & 0.000000 & & & & \\
\hline C & 0.000000 & 5.054470 & -1.207458 & & & & \\
\hline C & 0.000000 & 3.658518 & -1.207873 & & & & \\
\hline C & 0.000000 & -3.658518 & 1.207873 & & & & \\
\hline C & 0.000000 & -5.054470 & 1.207458 & & & & \\
\hline C & 0.000000 & -5.755998 & 0.000000 & & & & \\
\hline C & 0.000000 & -5.054470 & -1.207458 & & & & \\
\hline C & 0.000000 & -3.658518 & -1.207873 & & & & \\
\hline
\end{tabular}




\begin{tabular}{|c|c|c|c|c|c|c|c|}
\hline & $x$ & & 7 & & $x$ & , & 7 \\
\hline C & 7.547505 & 0.000000 & 0.000000 & $\mathrm{H}$ & 5.165091 & 3.401187 & 0.000000 \\
\hline C & 6.866373 & 1.221215 & 0.000000 & $\mathrm{H}$ & 5.165091 & -3.401187 & 0.000000 \\
\hline C & 5.455075 & 1.251896 & 0.000000 & $\mathrm{H}$ & -5.165091 & 3.401187 & 0.000000 \\
\hline C & 4.655877 & 2.440114 & 0.000000 & $\mathrm{H}$ & -5.165091 & -3.401187 & 0.000000 \\
\hline C & 3.264961 & 2.414524 & 0.000000 & $\mathrm{H}$ & 8.634172 & 0.000000 & 0.000000 \\
\hline C & 2.588908 & 1.174496 & 0.000000 & $\mathrm{H}$ & 7.425762 & 2.153752 & 0.000000 \\
\hline C & 3.380255 & 0.000000 & 0.000000 & $\mathrm{H}$ & 7.425762 & -2.153752 & 0.000000 \\
\hline C & 2.588908 & -1.174496 & 0.000000 & $\mathrm{H}$ & 2.716606 & 3.350095 & 0.000000 \\
\hline C & 3.264961 & -2.414524 & 0.000000 & $\mathrm{H}$ & 2.716606 & -3.350095 & 0.000000 \\
\hline C & 4.655877 & -2.440114 & 0.000000 & $\mathrm{H}$ & -2.716606 & 3.350095 & 0.000000 \\
\hline C & 5.455075 & -1.251896 & 0.000000 & $\mathrm{H}$ & -7.425762 & 2.153752 & 0.000000 \\
\hline C & 6.866373 & -1.221215 & 0.000000 & $\mathrm{H}$ & -8.634172 & 0.000000 & 0.000000 \\
\hline C & 4.774560 & 0.000000 & 0.000000 & $\mathrm{H}$ & -7.425762 & -2.153752 & 0.000000 \\
\hline C & 1.201061 & 0.723589 & 0.000000 & $\mathrm{H}$ & -2.716606 & -3.350095 & 0.000000 \\
\hline C & 1.201061 & -0.723589 & 0.000000 & $\mathrm{H}$ & 0.000000 & 3.112888 & 2.147470 \\
\hline C & 0.000000 & -1.449335 & 0.000000 & $\mathrm{H}$ & 0.000000 & 5.592948 & 2.151282 \\
\hline C & -1.201061 & -0.723589 & 0.000000 & $\mathrm{H}$ & 0.000000 & 6.842303 & 0.000000 \\
\hline C & -1.201061 & 0.723589 & 0.000000 & $\mathrm{H}$ & 0.000000 & 5.592948 & -2.151282 \\
\hline C & 0.000000 & 1.449335 & 0.000000 & $\mathrm{H}$ & 0.000000 & 3.112888 & -2.147470 \\
\hline C & -3.380255 & 0.000000 & 0.000000 & $\mathrm{H}$ & 0.000000 & -3.112888 & 2.147470 \\
\hline C & -2.588908 & 1.174496 & 0.000000 & $\mathrm{H}$ & 0.000000 & -5.592948 & 2.151282 \\
\hline C & -3.264961 & 2.414524 & 0.000000 & $\mathrm{H}$ & 0.000000 & -6.842303 & 0.000000 \\
\hline C & -4.655877 & 2.440114 & 0.000000 & $\mathrm{H}$ & 0.000000 & -5.592948 & -2.151282 \\
\hline C & -5.455075 & 1.251896 & 0.000000 & $\mathrm{H}$ & 0.000000 & -3.112888 & -2.147470 \\
\hline C & -6.866373 & 1.221215 & 0.000000 & & & & \\
\hline C & -7.547505 & 0.000000 & 0.000000 & & & & \\
\hline C & -6.866373 & -1.221215 & 0.000000 & & & & \\
\hline C & -5.455075 & -1.251896 & 0.000000 & & & & \\
\hline C & -4.655877 & -2.440114 & 0.000000 & & & & \\
\hline C & -3.264961 & -2.414524 & 0.000000 & & & & \\
\hline C & -2.588908 & -1.174496 & 0.000000 & & & & \\
\hline C & -4.774560 & 0.000000 & 0.000000 & & & & \\
\hline C & 0.000000 & 2.944626 & 0.000000 & & & & \\
\hline C & 0.000000 & -2.944626 & 0.000000 & & & & \\
\hline C & 0.000000 & 3.657990 & 1.207593 & & & & \\
\hline C & 0.000000 & 5.053996 & 1.207447 & & & & \\
\hline C & 0.000000 & 5.755555 & 0.000000 & & & & \\
\hline C & 0.000000 & 5.053996 & -1.207447 & & & & \\
\hline C & 0.000000 & 3.657990 & -1.207593 & & & & \\
\hline C & 0.000000 & -3.657990 & 1.207593 & & & & \\
\hline C & 0.000000 & -5.053996 & 1.207447 & & & & \\
\hline C & 0.000000 & -5.755555 & 0.000000 & & & & \\
\hline C & 0.000000 & -5.053996 & -1.207447 & & & & \\
\hline C & 0.000000 & -3.657990 & -1.207593 & & & & \\
\hline
\end{tabular}


2 (1-D chain, RB3LYP/6-31G*)

\begin{tabular}{|c|c|c|c|c|c|c|c|}
\hline & $x$ & $y$ & $z$ & & $x$ & $y$ & $z$ \\
\hline C & -5.021008 & 1.404504 & 0.037916 & $\mathrm{H}$ & -2.383668 & -3.348829 & 0.976042 \\
\hline C & -3.870618 & 0.695830 & 0.420435 & $\mathrm{H}$ & -0.056434 & -3.363089 & 1.739418 \\
\hline $\mathrm{C}$ & -3.855629 & -0.745143 & 0.443455 & $\mathrm{H}$ & 2.085422 & -2.086069 & 2.393346 \\
\hline C & -2.514820 & -1.179284 & 0.864949 & $\mathrm{H}$ & 3.219292 & 0.082332 & 2.716708 \\
\hline C & -1.873476 & -2.403856 & 1.126024 & $\mathrm{H}$ & 2.062816 & 2.215549 & 2.259900 \\
\hline C & -0.545073 & -2.409832 & 1.554042 & $\mathrm{H}$ & -0.100629 & 3.428191 & 1.556774 \\
\hline C & 0.194355 & -1.213031 & 1.795491 & $\mathrm{H}$ & -2.436633 & 3.343873 & 0.824749 \\
\hline $\mathrm{C}$ & 1.546164 & -1.161166 & 2.205857 & $\mathrm{H}$ & -4.756839 & 3.055304 & -2.106611 \\
\hline C & 2.181264 & 0.067141 & 2.397819 & $\mathrm{H}$ & -4.773189 & 5.535003 & -2.130501 \\
\hline $\mathrm{C}$ & 1.533573 & 1.275406 & 2.129471 & $\mathrm{H}$ & -5.081909 & 6.800004 & -0.011115 \\
\hline $\mathrm{C}$ & 0.178906 & 1.287591 & 1.726511 & $\mathrm{H}$ & -5.362801 & 5.564900 & 2.131093 \\
\hline C & -0.577017 & 2.460219 & 1.424029 & $\mathrm{H}$ & -5.323042 & 3.085763 & 2.149165 \\
\hline C & -1.911618 & 2.414620 & 1.015965 & $\mathrm{H}$ & -5.295356 & -3.137716 & 2.219792 \\
\hline $\mathrm{C}$ & -2.537286 & 1.170506 & 0.821541 & $\mathrm{H}$ & -5.281686 & -5.617220 & 2.246773 \\
\hline C & -1.784455 & 0.007266 & 1.110276 & $\mathrm{H}$ & -4.936146 & -6.884847 & 0.133690 \\
\hline C & -0.466973 & 0.027955 & 1.567230 & $\mathrm{H}$ & -4.615288 & -5.652189 & -2.003514 \\
\hline $\mathrm{C}$ & -4.989297 & -1.489252 & 0.079629 & $\mathrm{H}$ & -4.650841 & -3.172753 & -2.024557 \\
\hline $\mathrm{C}$ & 3.864949 & -0.687763 & -0.401950 & $\mathrm{H}$ & 2.360423 & 3.355349 & -0.917599 \\
\hline C & 3.848531 & 0.753250 & -0.421790 & $\mathrm{H}$ & 0.022455 & 3.366193 & -1.648765 \\
\hline $\mathrm{C}$ & 2.504632 & 1.186343 & -0.835584 & $\mathrm{H}$ & -2.118221 & 2.086318 & -2.301166 \\
\hline $\mathrm{C}$ & 1.853892 & 2.409240 & -1.072749 & $\mathrm{H}$ & -3.245495 & -0.083742 & -2.636045 \\
\hline C & 0.517498 & 2.413278 & -1.480101 & $\mathrm{H}$ & -2.078531 & -2.215638 & -2.199322 \\
\hline $\mathrm{C}$ & -0.217051 & 1.216137 & -1.731012 & $\mathrm{H}$ & 0.098094 & -3.424971 & -1.535144 \\
\hline C & -1.573669 & 1.162222 & -2.126331 & $\mathrm{H}$ & 2.438485 & -3.337074 & -0.817011 \\
\hline $\mathrm{C}$ & -2.204905 & -0.067265 & -2.326102 & TV & 10.003865 & 0.092900 & -0.097918 \\
\hline $\mathrm{C}$ & -1.552098 & -1.274130 & -2.067617 & & & & \\
\hline C & -0.190803 & -1.284696 & -1.683178 & & & & \\
\hline C & 0.572613 & -2.456133 & -1.401458 & & & & \\
\hline $\mathrm{C}$ & 1.909186 & -2.408447 & -0.999016 & & & & \\
\hline $\mathrm{C}$ & 2.532477 & -1.163564 & -0.803850 & & & & \\
\hline $\mathrm{C}$ & 1.774813 & -0.001630 & -1.080588 & & & & \\
\hline C & 0.452569 & -0.023892 & -1.522995 & & & & \\
\hline $\mathrm{C}$ & 4.965369 & 2.992160 & -0.075079 & & & & \\
\hline $\mathrm{C}$ & 5.121415 & 3.699566 & -1.276412 & & & & \\
\hline C & 5.107168 & 5.095565 & -1.288841 & & & & \\
\hline $\mathrm{C}$ & 4.933793 & 5.806279 & -0.099280 & & & & \\
\hline $\mathrm{C}$ & 4.776236 & 5.112744 & 1.102537 & & & & \\
\hline C & 4.793445 & 3.716864 & 1.113833 & & & & \\
\hline C & 5.030551 & -2.891201 & -0.001749 & & & & \\
\hline $\mathrm{C}$ & 4.851208 & -3.597330 & 1.196985 & & & & \\
\hline $\mathrm{C}$ & 4.863546 & -4.993310 & 1.211048 & & & & \\
\hline C & 5.057537 & -5.705312 & 0.025426 & & & & \\
\hline C & 5.237706 & -5.013011 & -1.173919 & & & & \\
\hline $\mathrm{C}$ & 5.222898 & -3.617107 & -1.186751 & & & & \\
\hline
\end{tabular}




\begin{tabular}{|c|c|c|c|c|c|c|c|}
\hline & $x$ & v & 7 & & $x$ & v & 7 \\
\hline C & 7.549076 & 0.000000 & 0.000000 & $\mathrm{H}$ & 5.163494 & 3.396367 & 0.000000 \\
\hline C & 6.865060 & 1.219489 & 0.000000 & $\mathrm{H}$ & 5.163494 & -3.396367 & 0.000000 \\
\hline C & 5.451171 & 1.249196 & 0.000000 & $\mathrm{H}$ & -5.163494 & 3.396367 & 0.000000 \\
\hline C & 4.656331 & 2.434883 & 0.000000 & $\mathrm{H}$ & -5.163494 & -3.396367 & 0.000000 \\
\hline C & 3.259853 & 2.412287 & 0.000000 & $\mathrm{H}$ & 8.634501 & 0.000000 & 0.000000 \\
\hline C & 2.592856 & 1.173101 & 0.000000 & $\mathrm{H}$ & 7.424205 & 2.151564 & 0.000000 \\
\hline C & 3.382370 & 0.000000 & 0.000000 & $\mathrm{H}$ & 7.424205 & -2.151564 & 0.000000 \\
\hline C & 2.592856 & -1.173101 & 0.000000 & $\mathrm{H}$ & 2.712553 & 3.347504 & 0.000000 \\
\hline C & 3.259853 & -2.412287 & 0.000000 & $\mathrm{H}$ & 2.712553 & -3.347504 & 0.000000 \\
\hline C & 4.656331 & -2.434883 & 0.000000 & $\mathrm{H}$ & -2.712553 & 3.347504 & 0.000000 \\
\hline C & 5.451171 & -1.249196 & 0.000000 & $\mathrm{H}$ & -7.424205 & 2.151564 & 0.000000 \\
\hline C & 6.865060 & -1.219489 & 0.000000 & $\mathrm{H}$ & -8.634501 & 0.000000 & 0.000000 \\
\hline C & 4.773470 & 0.000000 & 0.000000 & $\mathrm{H}$ & -7.424205 & -2.151564 & 0.000000 \\
\hline C & 1.196372 & 0.718637 & 0.000000 & $\mathrm{H}$ & -2.712553 & -3.347504 & 0.000000 \\
\hline C & 1.196372 & -0.718637 & 0.000000 & $\mathrm{H}$ & 0.000000 & 3.114492 & 2.151513 \\
\hline C & 0.000000 & -1.454076 & 0.000000 & $\mathrm{H}$ & 0.000000 & 5.593111 & 2.151463 \\
\hline C & -1.196372 & -0.718637 & 0.000000 & $\mathrm{H}$ & 0.000000 & 6.840194 & 0.000000 \\
\hline C & -1.196372 & 0.718637 & 0.000000 & $\mathrm{H}$ & 0.000000 & 5.593111 & -2.151463 \\
\hline C & 0.000000 & 1.454076 & 0.000000 & $\mathrm{H}$ & 0.000000 & 3.114492 & -2.151513 \\
\hline C & -3.382370 & 0.000000 & 0.000000 & $\mathrm{H}$ & 0.000000 & -3.114492 & 2.151513 \\
\hline C & -2.592856 & 1.173101 & 0.000000 & $\mathrm{H}$ & 0.000000 & -5.593111 & 2.151463 \\
\hline C & -3.259853 & 2.412287 & 0.000000 & $\mathrm{H}$ & 0.000000 & -6.840194 & 0.000000 \\
\hline C & -4.656331 & 2.434883 & 0.000000 & $\mathrm{H}$ & 0.000000 & -5.593111 & -2.151463 \\
\hline C & -5.451171 & 1.249196 & 0.000000 & $\mathrm{H}$ & 0.000000 & -3.114492 & -2.151513 \\
\hline C & -6.865060 & 1.219489 & 0.000000 & & & & \\
\hline C & -7.549076 & 0.000000 & 0.000000 & & & & \\
\hline C & -6.865060 & -1.219489 & 0.000000 & & & & \\
\hline C & -5.451171 & -1.249196 & 0.000000 & & & & \\
\hline C & -4.656331 & -2.434883 & 0.000000 & & & & \\
\hline C & -3.259853 & -2.412287 & 0.000000 & & & & \\
\hline C & -2.592856 & -1.173101 & 0.000000 & & & & \\
\hline C & -4.773470 & 0.000000 & 0.000000 & & & & \\
\hline C & 0.000000 & 2.948166 & 0.000000 & & & & \\
\hline C & 0.000000 & -2.948166 & 0.000000 & & & & \\
\hline C & 0.000000 & 3.657538 & 1.210277 & & & & \\
\hline C & 0.000000 & 5.053723 & 1.208446 & & & & \\
\hline C & 0.000000 & 5.753959 & 0.000000 & & & & \\
\hline C & 0.000000 & 5.053723 & -1.208446 & & & & \\
\hline C & 0.000000 & 3.657538 & -1.210277 & & & & \\
\hline C & 0.000000 & -3.657538 & 1.210277 & & & & \\
\hline C & 0.000000 & -5.053723 & 1.208446 & & & & \\
\hline C & 0.000000 & -5.753959 & 0.000000 & & & & \\
\hline C & 0.000000 & -5.053723 & -1.208446 & & & & \\
\hline C & 0.000000 & -3.657538 & -1.210277 & & & & \\
\hline
\end{tabular}


2 (dication, RB3LYP/6-31G*, $D_{2 h}$ )

\begin{tabular}{|c|c|c|c|c|c|c|c|}
\hline & $x$ & $y$ & $z$ & & $x$ & $y$ & $z$ \\
\hline C & 7.562627 & 0.000000 & 0.000000 & $\mathrm{H}$ & 5.171916 & 3.392371 & 0.000000 \\
\hline C & 6.874860 & 1.217704 & 0.000000 & $\mathrm{H}$ & 5.171916 & -3.392371 & 0.000000 \\
\hline C & 5.455579 & 1.246972 & 0.000000 & $\mathrm{H}$ & -5.171916 & 3.392371 & 0.000000 \\
\hline C & 4.667612 & 2.429458 & 0.000000 & $\mathrm{H}$ & -5.171916 & -3.392371 & 0.000000 \\
\hline C & 3.260578 & 2.408004 & 0.000000 & $\mathrm{H}$ & 8.647413 & 0.000000 & 0.000000 \\
\hline C & 2.604955 & 1.172565 & 0.000000 & $\mathrm{H}$ & 7.434131 & 2.149824 & 0.000000 \\
\hline C & 3.391626 & 0.000000 & 0.000000 & $\mathrm{H}$ & 7.434131 & -2.149824 & 0.000000 \\
\hline C & 2.604955 & -1.172565 & 0.000000 & $\mathrm{H}$ & 2.714160 & 3.343365 & 0.000000 \\
\hline C & 3.260578 & -2.408004 & 0.000000 & $\mathrm{H}$ & 2.714160 & -3.343365 & 0.000000 \\
\hline C & 4.667612 & -2.429458 & 0.000000 & $\mathrm{H}$ & -2.714160 & 3.343365 & 0.000000 \\
\hline C & 5.455579 & -1.246972 & 0.000000 & $\mathrm{H}$ & -7.434131 & 2.149824 & 0.000000 \\
\hline C & 6.874860 & -1.217704 & 0.000000 & $\mathrm{H}$ & -8.647413 & 0.000000 & 0.000000 \\
\hline C & 4.779606 & 0.000000 & 0.000000 & $\mathrm{H}$ & -7.434131 & -2.149824 & 0.000000 \\
\hline C & 1.190787 & 0.712459 & 0.000000 & $\mathrm{H}$ & -2.714160 & -3.343365 & 0.000000 \\
\hline C & 1.190787 & -0.712459 & 0.000000 & $\mathrm{H}$ & 0.000000 & 3.117150 & 2.155427 \\
\hline C & 0.000000 & -1.457508 & 0.000000 & $\mathrm{H}$ & 0.000000 & 5.594532 & 2.151752 \\
\hline C & -1.190787 & -0.712459 & 0.000000 & $\mathrm{H}$ & 0.000000 & 6.839753 & 0.000000 \\
\hline C & -1.190787 & 0.712459 & 0.000000 & $\mathrm{H}$ & 0.000000 & 5.594532 & -2.151752 \\
\hline C & 0.000000 & 1.457508 & 0.000000 & $\mathrm{H}$ & 0.000000 & 3.117150 & -2.155427 \\
\hline C & -3.391626 & 0.000000 & 0.000000 & $\mathrm{H}$ & 0.000000 & -3.117150 & 2.155427 \\
\hline C & -2.604955 & 1.172565 & 0.000000 & $\mathrm{H}$ & 0.000000 & -5.594532 & 2.151752 \\
\hline C & -3.260578 & 2.408004 & 0.000000 & $\mathrm{H}$ & 0.000000 & -6.839753 & 0.000000 \\
\hline C & -4.667612 & 2.429458 & 0.000000 & $\mathrm{H}$ & 0.000000 & -5.594532 & -2.151752 \\
\hline C & -5.455579 & 1.246972 & 0.000000 & $\mathrm{H}$ & 0.000000 & -3.117150 & -2.155427 \\
\hline C & -6.874860 & 1.217704 & 0.000000 & & & & \\
\hline C & -7.562627 & 0.000000 & 0.000000 & & & & \\
\hline C & -6.874860 & -1.217704 & 0.000000 & & & & \\
\hline C & -5.455579 & -1.246972 & 0.000000 & & & & \\
\hline C & -4.667612 & -2.429458 & 0.000000 & & & & \\
\hline C & -3.260578 & -2.408004 & 0.000000 & & & & \\
\hline C & -2.604955 & -1.172565 & 0.000000 & & & & \\
\hline C & -4.779606 & 0.000000 & 0.000000 & & & & \\
\hline C & 0.000000 & 2.951147 & 0.000000 & & & & \\
\hline C & 0.000000 & -2.951147 & 0.000000 & & & & \\
\hline C & 0.000000 & 3.657943 & 1.212703 & & & & \\
\hline C & 0.000000 & 5.054550 & 1.209445 & & & & \\
\hline C & 0.000000 & 5.753855 & 0.000000 & & & & \\
\hline C & 0.000000 & 5.054550 & -1.209445 & & & & \\
\hline C & 0.000000 & 3.657943 & -1.212703 & & & & \\
\hline C & 0.000000 & -3.657943 & 1.212703 & & & & \\
\hline C & 0.000000 & -5.054550 & 1.209445 & & & & \\
\hline C & 0.000000 & -5.753855 & 0.000000 & & & & \\
\hline C & 0.000000 & -5.054550 & -1.209445 & & & & \\
\hline C & 0.000000 & -3.657943 & -1.212703 & & & & \\
\hline
\end{tabular}


2 (dianion, RB3LYP/6-31G*, $D_{2 h}$ )

\begin{tabular}{|c|c|c|c|c|c|c|c|}
\hline & $x$ & $y$ & $z$ & & $x$ & $\mathrm{y}$ & $z$ \\
\hline C & 7.574593 & 0.000000 & 0.000000 & $\mathrm{H}$ & 5.189408 & 3.410588 & 0.000000 \\
\hline C & 6.901675 & 1.225361 & 0.000000 & $\mathrm{H}$ & 5.189408 & -3.410588 & 0.000000 \\
\hline C & 5.484899 & 1.261288 & 0.000000 & $\mathrm{H}$ & -5.189408 & 3.410588 & 0.000000 \\
\hline C & 4.682234 & 2.445248 & 0.000000 & $\mathrm{H}$ & -5.189408 & -3.410588 & 0.000000 \\
\hline C & 3.288044 & 2.409068 & 0.000000 & $\mathrm{H}$ & 8.666692 & 0.000000 & 0.000000 \\
\hline C & 2.600859 & 1.178953 & 0.000000 & $\mathrm{H}$ & 7.463576 & 2.159660 & 0.000000 \\
\hline C & 3.394694 & 0.000000 & 0.000000 & $\mathrm{H}$ & 7.463576 & -2.159660 & 0.000000 \\
\hline C & 2.600859 & -1.178953 & 0.000000 & $\mathrm{H}$ & 2.742156 & 3.349776 & 0.000000 \\
\hline C & 3.288044 & -2.409068 & 0.000000 & $\mathrm{H}$ & 2.742156 & -3.349776 & 0.000000 \\
\hline C & 4.682234 & -2.445248 & 0.000000 & $\mathrm{H}$ & -2.742156 & 3.349776 & 0.000000 \\
\hline C & 5.484899 & -1.261288 & 0.000000 & $\mathrm{H}$ & -7.463576 & 2.159660 & 0.000000 \\
\hline C & 6.901675 & -1.225361 & 0.000000 & $\mathrm{H}$ & -8.666692 & 0.000000 & 0.000000 \\
\hline C & 4.797241 & 0.000000 & 0.000000 & $\mathrm{H}$ & -7.463576 & -2.159660 & 0.000000 \\
\hline C & 1.215013 & 0.728918 & 0.000000 & $\mathrm{H}$ & -2.742156 & -3.349776 & 0.000000 \\
\hline C & 1.215013 & -0.728918 & 0.000000 & $\mathrm{H}$ & 0.000000 & 3.109801 & 2.140449 \\
\hline C & 0.000000 & -1.439457 & 0.000000 & $\mathrm{H}$ & 0.000000 & 5.593294 & 2.151735 \\
\hline C & -1.215013 & -0.728918 & 0.000000 & $\mathrm{H}$ & 0.000000 & 6.848553 & 0.000000 \\
\hline C & -1.215013 & 0.728918 & 0.000000 & $\mathrm{H}$ & 0.000000 & 5.593294 & -2.151735 \\
\hline C & 0.000000 & 1.439457 & 0.000000 & $\mathrm{H}$ & 0.000000 & 3.109801 & -2.140449 \\
\hline C & -3.394694 & 0.000000 & 0.000000 & $\mathrm{H}$ & 0.000000 & -3.109801 & 2.140449 \\
\hline C & -2.600859 & 1.178953 & 0.000000 & $\mathrm{H}$ & 0.000000 & -5.593294 & 2.151735 \\
\hline C & -3.288044 & 2.409068 & 0.000000 & $\mathrm{H}$ & 0.000000 & -6.848553 & 0.000000 \\
\hline C & -4.682234 & 2.445248 & 0.000000 & $\mathrm{H}$ & 0.000000 & -5.593294 & -2.151735 \\
\hline C & -5.484899 & 1.261288 & 0.000000 & $\mathrm{H}$ & 0.000000 & -3.109801 & -2.140449 \\
\hline C & -6.901675 & 1.225361 & 0.000000 & & & & \\
\hline C & -7.574593 & 0.000000 & 0.000000 & & & & \\
\hline C & -6.901675 & -1.225361 & 0.000000 & & & & \\
\hline C & -5.484899 & -1.261288 & 0.000000 & & & & \\
\hline C & -4.682234 & -2.445248 & 0.000000 & & & & \\
\hline C & -3.288044 & -2.409068 & 0.000000 & & & & \\
\hline C & -2.600859 & -1.178953 & 0.000000 & & & & \\
\hline C & -4.797241 & 0.000000 & 0.000000 & & & & \\
\hline C & 0.000000 & 2.934220 & 0.000000 & & & & \\
\hline C & 0.000000 & -2.934220 & 0.000000 & & & & \\
\hline C & 0.000000 & 3.658536 & 1.202604 & & & & \\
\hline C & 0.000000 & 5.054605 & 1.205542 & & & & \\
\hline C & 0.000000 & 5.759798 & 0.000000 & & & & \\
\hline C & 0.000000 & 5.054605 & -1.205542 & & & & \\
\hline C & 0.000000 & 3.658536 & -1.202604 & & & & \\
\hline C & 0.000000 & -3.658536 & 1.202604 & & & & \\
\hline C & 0.000000 & -5.054605 & 1.205542 & & & & \\
\hline C & 0.000000 & -5.759798 & 0.000000 & & & & \\
\hline C & 0.000000 & -5.054605 & -1.205542 & & & & \\
\hline C & 0.000000 & -3.658536 & -1.202604 & & & & \\
\hline
\end{tabular}




\begin{tabular}{|c|c|c|c|c|c|c|c|}
\hline & $x$ & $y$ & $z$ & & & & \\
\hline C & -7.516565 & 0.000000 & 0.000000 & $\mathrm{H}$ & -5.486743 & 4.055605 & 2.171123 \\
\hline C & -6.843982 & 1.219625 & 0.000000 & $\mathrm{H}$ & -6.933331 & 3.406104 & 1.406139 \\
\hline C & -5.433809 & 1.275162 & 0.000000 & $\mathrm{H}$ & -6.569551 & 5.129897 & 1.269020 \\
\hline C & -4.628179 & 2.502336 & 0.000000 & $\mathrm{H}$ & -6.933331 & 3.406104 & -1.406139 \\
\hline C & -3.238144 & 2.408597 & 0.000000 & $\mathrm{H}$ & -5.486743 & 4.055605 & -2.171123 \\
\hline C & -2.578553 & 1.165549 & 0.000000 & $\mathrm{H}$ & -6.569551 & 5.129897 & -1.269020 \\
\hline C & -3.371854 & 0.000000 & 0.000000 & $\mathrm{H}$ & -3.587835 & 5.018754 & -0.889097 \\
\hline C & -2.578553 & -1.165549 & 0.000000 & $\mathrm{H}$ & -3.587835 & 5.018754 & 0.889097 \\
\hline C & -3.238144 & -2.408597 & 0.000000 & $\mathrm{H}$ & -4.746810 & 6.009337 & 0.000000 \\
\hline C & -4.628179 & -2.502336 & 0.000000 & $\mathrm{H}$ & -5.486743 & -4.055605 & -2.171123 \\
\hline $\mathrm{C}$ & -5.433809 & -1.275162 & 0.000000 & $\mathrm{H}$ & -6.933331 & -3.406104 & -1.406139 \\
\hline C & -6.843982 & -1.219625 & 0.000000 & $\mathrm{H}$ & -6.569551 & -5.129897 & -1.269020 \\
\hline C & -4.763959 & 0.000000 & 0.000000 & $\mathrm{H}$ & -6.933331 & -3.406104 & 1.406139 \\
\hline $\mathrm{C}$ & -1.201544 & 0.725644 & 0.000000 & $\mathrm{H}$ & -5.486743 & -4.055605 & 2.171123 \\
\hline C & -1.201544 & -0.725644 & 0.000000 & $\mathrm{H}$ & -6.569551 & -5.129897 & 1.269020 \\
\hline C & 0.000000 & -1.436827 & 0.000000 & $\mathrm{H}$ & -3.587835 & -5.018754 & 0.889097 \\
\hline C & 1.201544 & -0.725644 & 0.000000 & $\mathrm{H}$ & -3.587835 & -5.018754 & -0.889097 \\
\hline $\mathrm{C}$ & 1.201544 & 0.725644 & 0.000000 & $\mathrm{H}$ & -4.746810 & -6.009337 & 0.000000 \\
\hline $\mathrm{C}$ & 0.000000 & 1.436827 & 0.000000 & $\mathrm{H}$ & 5.486743 & 4.055605 & -2.171123 \\
\hline C & 3.371854 & 0.000000 & 0.000000 & $\mathrm{H}$ & 6.933331 & 3.406104 & -1.406139 \\
\hline C & 2.578553 & 1.165549 & 0.000000 & $\mathrm{H}$ & 6.569551 & 5.129897 & -1.269020 \\
\hline $\mathrm{C}$ & 3.238144 & 2.408597 & 0.000000 & $\mathrm{H}$ & 6.933331 & 3.406104 & 1.406139 \\
\hline $\mathrm{C}$ & 4.628179 & 2.502336 & 0.000000 & $\mathrm{H}$ & 5.486743 & 4.055605 & 2.171123 \\
\hline C & 5.433809 & 1.275162 & 0.000000 & $\mathrm{H}$ & 6.569551 & 5.129897 & 1.269020 \\
\hline $\mathrm{C}$ & 6.843982 & 1.219625 & 0.000000 & $\mathrm{H}$ & 3.587835 & 5.018754 & 0.889097 \\
\hline $\mathrm{C}$ & 7.516565 & 0.000000 & 0.000000 & $\mathrm{H}$ & 3.587835 & 5.018754 & -0.889097 \\
\hline C & 6.843982 & -1.219625 & 0.000000 & $\mathrm{H}$ & 4.746810 & 6.009337 & 0.000000 \\
\hline C & 5.433809 & -1.275162 & 0.000000 & $\mathrm{H}$ & 5.486743 & -4.055605 & 2.171123 \\
\hline $\mathrm{C}$ & 4.628179 & -2.502336 & 0.000000 & $\mathrm{H}$ & 6.933331 & -3.406104 & 1.406139 \\
\hline $\mathrm{C}$ & 3.238144 & -2.408597 & 0.000000 & $\mathrm{H}$ & 6.569551 & -5.129897 & 1.269020 \\
\hline $\mathrm{C}$ & 2.578553 & -1.165549 & 0.000000 & $\mathrm{H}$ & 6.933331 & -3.406104 & -1.406139 \\
\hline C & 4.763959 & 0.000000 & 0.000000 & $\mathrm{H}$ & 5.486743 & -4.055605 & -2.171123 \\
\hline $\mathrm{C}$ & -5.278029 & 3.909910 & 0.000000 & $\mathrm{H}$ & 6.569551 & -5.129897 & -1.269020 \\
\hline $\mathrm{C}$ & -6.124447 & 4.127462 & 1.282653 & $\mathrm{H}$ & 3.587835 & -5.018754 & -0.889097 \\
\hline C & -6.124447 & 4.127462 & -1.282653 & $\mathrm{H}$ & 3.587835 & -5.018754 & 0.889097 \\
\hline $\mathrm{C}$ & -4.227267 & 5.044704 & 0.000000 & $\mathrm{H}$ & 4.746810 & -6.009337 & 0.000000 \\
\hline C & -5.278029 & -3.909910 & 0.000000 & $\mathrm{H}$ & -8.603356 & 0.000000 & 0.000000 \\
\hline C & -6.124447 & -4.127462 & -1.282653 & $\mathrm{H}$ & -7.439794 & 2.120317 & 0.000000 \\
\hline C & -6.124447 & -4.127462 & 1.282653 & $\mathrm{H}$ & -7.439794 & -2.120317 & 0.000000 \\
\hline $\mathrm{C}$ & -4.227267 & -5.044704 & 0.000000 & $\mathrm{H}$ & -2.639483 & 3.310287 & 0.000000 \\
\hline C & 5.278029 & 3.909910 & 0.000000 & $\mathrm{H}$ & -2.639483 & -3.310287 & 0.000000 \\
\hline $\mathrm{C}$ & 6.124447 & 4.127462 & -1.282653 & $\mathrm{H}$ & 0.000000 & 2.525253 & 0.000000 \\
\hline $\mathrm{C}$ & 6.124447 & 4.127462 & 1.282653 & $\mathrm{H}$ & 0.000000 & -2.525253 & 0.000000 \\
\hline C & 4.227267 & 5.044704 & 0.000000 & $\mathrm{H}$ & 2.639483 & 3.310287 & 0.000000 \\
\hline C & 5.278029 & -3.909910 & 0.000000 & $\mathrm{H}$ & 7.439794 & 2.120317 & 0.000000 \\
\hline C & 6.124447 & -4.127462 & 1.282653 & $\mathrm{H}$ & 8.603356 & 0.000000 & 0.000000 \\
\hline $\mathrm{C}$ & 6.124447 & -4.127462 & -1.282653 & $\mathrm{H}$ & 7.439794 & -2.120317 & 0.000000 \\
\hline $\mathrm{C}$ & 4.227267 & 704 & 0.000000 & $\mathrm{H}$ & 2.639483 & -3.310287 & 0.000000 \\
\hline
\end{tabular}




\begin{tabular}{|c|c|c|c|c|c|c|c|}
\hline & $x$ & V & 7 & & & , & \\
\hline C & -7.523729 & 0.000000 & 0.000000 & $\mathrm{H}$ & -5.492514 & 4.055032 & 2.171178 \\
\hline C & -6.851782 & 1.219860 & 0.000000 & $\mathrm{H}$ & -6.939717 & 3.407451 & 1.405744 \\
\hline C & -5.439318 & 1.275903 & 0.000000 & $\mathrm{H}$ & -6.573731 & 5.130797 & 1.268958 \\
\hline C & -4.635872 & 2.500269 & 0.000000 & $\mathrm{H}$ & -6.939717 & 3.407451 & -1.405744 \\
\hline C & -3.240780 & 2.405061 & 0.000000 & $\mathrm{H}$ & -5.492514 & 4.055032 & -2.171178 \\
\hline C & -2.586000 & 1.165360 & 0.000000 & $\mathrm{H}$ & -6.573731 & 5.130797 & -1.268958 \\
\hline C & -3.377987 & 0.000000 & 0.000000 & $\mathrm{H}$ & -3.591805 & 5.015699 & -0.889112 \\
\hline C & -2.586000 & -1.165360 & 0.000000 & $\mathrm{H}$ & -3.591805 & 5.015699 & 0.889112 \\
\hline C & -3.240780 & -2.405061 & 0.000000 & $\mathrm{H}$ & -4.749443 & 6.007670 & 0.000000 \\
\hline C & -4.635872 & -2.500269 & 0.000000 & $\mathrm{H}$ & -5.492514 & -4.055032 & -2.171178 \\
\hline C & -5.439318 & -1.275903 & 0.000000 & $\mathrm{H}$ & -6.939717 & -3.407451 & -1.405744 \\
\hline $\mathrm{C}$ & -6.851782 & -1.219860 & 0.000000 & $\mathrm{H}$ & -6.573731 & -5.130797 & -1.268958 \\
\hline $\mathrm{C}$ & -4.769933 & 0.000000 & 0.000000 & $\mathrm{H}$ & -6.939717 & -3.407451 & 1.405744 \\
\hline C & -1.200073 & 0.722294 & 0.000000 & $\mathrm{H}$ & -5.492514 & -4.055032 & 2.171178 \\
\hline C & -1.200073 & -0.722294 & 0.000000 & $\mathrm{H}$ & -6.573731 & -5.130797 & 1.268958 \\
\hline $\mathrm{C}$ & 0.000000 & -1.435370 & 0.000000 & $\mathrm{H}$ & -3.591805 & -5.015699 & 0.889112 \\
\hline $\mathrm{C}$ & 1.200073 & -0.722294 & 0.000000 & $\mathrm{H}$ & -3.591805 & -5.015699 & -0.889112 \\
\hline C & 1.200073 & 0.722294 & 0.000000 & $\mathrm{H}$ & -4.749443 & -6.007670 & 0.000000 \\
\hline C & 0.000000 & 1.435370 & 0.000000 & $\mathrm{H}$ & 5.492514 & 4.055032 & -2.171178 \\
\hline C & 3.377987 & 0.000000 & 0.000000 & $\mathrm{H}$ & 6.939717 & 3.407451 & -1.405744 \\
\hline C & 2.586000 & 1.165360 & 0.000000 & $\mathrm{H}$ & 6.573731 & 5.130797 & -1.268958 \\
\hline $\mathrm{C}$ & 3.240780 & 2.405061 & 0.000000 & $\mathrm{H}$ & 6.939717 & 3.407451 & 1.405744 \\
\hline $\mathrm{C}$ & 4.635872 & 2.500269 & 0.000000 & $\mathrm{H}$ & 5.492514 & 4.055032 & 2.171178 \\
\hline $\mathrm{C}$ & 5.439318 & 1.275903 & 0.000000 & $\mathrm{H}$ & 6.573731 & 5.130797 & 1.268958 \\
\hline C & 6.851782 & 1.219860 & 0.000000 & $\mathrm{H}$ & 3.591805 & 5.015699 & 0.889112 \\
\hline $\mathrm{C}$ & 7.523729 & 0.000000 & 0.000000 & $\mathrm{H}$ & 3.591805 & 5.015699 & -0.889112 \\
\hline $\mathrm{C}$ & 6.851782 & -1.219860 & 0.000000 & $\mathrm{H}$ & 4.749443 & 6.007670 & 0.000000 \\
\hline $\mathrm{C}$ & 5.439318 & -1.275903 & 0.000000 & $\mathrm{H}$ & 5.492514 & -4.055032 & 2.171178 \\
\hline $\mathrm{C}$ & 4.635872 & -2.500269 & 0.000000 & $\mathrm{H}$ & 6.939717 & -3.407451 & 1.405744 \\
\hline C & 3.240780 & -2.405061 & 0.000000 & $\mathrm{H}$ & 6.573731 & -5.130797 & 1.268958 \\
\hline C & 2.586000 & -1.165360 & 0.000000 & $\mathrm{H}$ & 6.939717 & -3.407451 & -1.405744 \\
\hline $\mathrm{C}$ & 4.769933 & 0.000000 & 0.000000 & $\mathrm{H}$ & 5.492514 & -4.055032 & -2.171178 \\
\hline $\mathrm{C}$ & -5.283671 & 3.908948 & 0.000000 & $\mathrm{H}$ & 6.573731 & -5.130797 & -1.268958 \\
\hline $\mathrm{C}$ & -6.129847 & 4.127803 & 1.282488 & $\mathrm{H}$ & 3.591805 & -5.015699 & -0.889112 \\
\hline C & -6.129847 & 4.127803 & -1.282488 & $\mathrm{H}$ & 3.591805 & -5.015699 & 0.889112 \\
\hline C & -4.231231 & 5.042310 & 0.000000 & $\mathrm{H}$ & 4.749443 & -6.007670 & 0.000000 \\
\hline$C$ & -5.283671 & -3.908948 & 0.000000 & $\mathrm{H}$ & -8.610566 & 0.000000 & 0.000000 \\
\hline C & -6.129847 & -4.127803 & -1.282488 & $\mathrm{H}$ & -7.447526 & 2.120557 & 0.000000 \\
\hline$C$ & -6.129847 & -4.127803 & 1.282488 & $\mathrm{H}$ & -7.447526 & -2.120557 & 0.000000 \\
\hline C & -4.231231 & -5.042310 & 0.000000 & $\mathrm{H}$ & -2.642500 & 3.307041 & 0.000000 \\
\hline$C$ & 5.283671 & 3.908948 & 0.000000 & $\mathrm{H}$ & -2.642500 & -3.307041 & 0.000000 \\
\hline$C$ & 6.129847 & 4.127803 & -1.282488 & $\mathrm{H}$ & 0.000000 & 2.523462 & 0.000000 \\
\hline$C$ & 6.129847 & 4.127803 & 1.282488 & $\mathrm{H}$ & 0.000000 & -2.523462 & 0.000000 \\
\hline $\mathrm{C}$ & 4.231231 & 5.042310 & 0.000000 & $\mathrm{H}$ & 2.642500 & 3.307041 & 0.000000 \\
\hline C & 5.283671 & -3.908948 & 0.000000 & $\mathrm{H}$ & 7.447526 & 2.120557 & 0.000000 \\
\hline$C$ & 6.129847 & -4.127803 & 1.282488 & $\mathrm{H}$ & 8.610566 & 0.000000 & 0.000000 \\
\hline C & 6.129847 & -4.127803 & -1.282488 & $\mathrm{H}$ & 7.447526 & -2.120557 & 0.000000 \\
\hline$C$ & 4.231231 & -5.042310 & 0.000000 & $\mathrm{H}$ & 2.642500 & -3.307041 & 0.000000 \\
\hline
\end{tabular}




\begin{tabular}{|c|c|c|c|c|c|c|c|}
\hline & $x$ & $\mathrm{v}$ & 7 & & $x$ & v & 7 \\
\hline$C$ & 5.436501 & 1.275685 & 0.000000 & $\mathrm{H}$ & 6.521980 & 1.342559 & 0.000000 \\
\hline C & 4.668239 & 2.426624 & 0.000000 & $\mathrm{H}$ & 6.521980 & -1.342559 & 0.000000 \\
\hline C & 3.246469 & 2.398136 & 0.000000 & $\mathrm{H}$ & -6.521980 & 1.342559 & 0.000000 \\
\hline C & 2.602866 & 1.172836 & 0.000000 & $\mathrm{H}$ & -6.521980 & -1.342559 & 0.000000 \\
\hline C & 3.400659 & 0.000000 & 0.000000 & $\mathrm{H}$ & 5.163885 & 3.394010 & 0.000000 \\
\hline C & 2.602866 & -1.172836 & 0.000000 & $\mathrm{H}$ & 5.163885 & -3.394010 & 0.000000 \\
\hline C & 3.246469 & -2.398136 & 0.000000 & $\mathrm{H}$ & -5.163885 & 3.394010 & 0.000000 \\
\hline C & 4.668239 & -2.426624 & 0.000000 & $\mathrm{H}$ & -5.163885 & -3.394010 & 0.000000 \\
\hline C & 5.436501 & -1.275685 & 0.000000 & $\mathrm{H}$ & 2.697562 & 3.333206 & 0.000000 \\
\hline C & 4.805262 & 0.000000 & 0.000000 & $\mathrm{H}$ & 2.697562 & -3.333206 & 0.000000 \\
\hline C & 1.197507 & 0.715874 & 0.000000 & $\mathrm{H}$ & -2.697562 & 3.333206 & 0.000000 \\
\hline C & 1.197507 & -0.715874 & 0.000000 & $\mathrm{H}$ & -2.697562 & -3.333206 & 0.000000 \\
\hline C & 0.000000 & -1.445929 & 0.000000 & $\mathrm{H}$ & 0.000000 & 3.110793 & 2.147286 \\
\hline C & -1.197507 & -0.715874 & 0.000000 & $\mathrm{H}$ & 0.000000 & 5.590755 & 2.151265 \\
\hline C & -1.197507 & 0.715874 & 0.000000 & $\mathrm{H}$ & 0.000000 & 6.840189 & 0.000000 \\
\hline C & 0.000000 & 1.445929 & 0.000000 & $\mathrm{H}$ & 0.000000 & 5.590755 & -2.151265 \\
\hline C & -3.400659 & 0.000000 & 0.000000 & $\mathrm{H}$ & 0.000000 & 3.110793 & -2.147286 \\
\hline C & -2.602866 & 1.172836 & 0.000000 & $\mathrm{H}$ & 0.000000 & -3.110793 & 2.147286 \\
\hline C & -3.246469 & 2.398136 & 0.000000 & $\mathrm{H}$ & 0.000000 & -5.590755 & 2.151265 \\
\hline C & -4.668239 & 2.426624 & 0.000000 & $\mathrm{H}$ & 0.000000 & -6.840189 & 0.000000 \\
\hline C & -5.436501 & 1.275685 & 0.000000 & $\mathrm{H}$ & 0.000000 & -5.590755 & -2.151265 \\
\hline C & -5.436501 & -1.275685 & 0.000000 & $\mathrm{H}$ & 0.000000 & -3.110793 & -2.147286 \\
\hline C & -4.668239 & -2.426624 & 0.000000 & & & & \\
\hline C & -3.246469 & -2.398136 & 0.000000 & & & & \\
\hline C & -2.602866 & -1.172836 & 0.000000 & & & & \\
\hline C & -4.805262 & 0.000000 & 0.000000 & & & & \\
\hline C & 0.000000 & 2.941399 & 0.000000 & & & & \\
\hline C & 0.000000 & -2.941399 & 0.000000 & & & & \\
\hline C & 0.000000 & 3.655735 & 1.207224 & & & & \\
\hline C & 0.000000 & 5.051758 & 1.207415 & & & & \\
\hline C & 0.000000 & 5.753410 & 0.000000 & & & & \\
\hline C & 0.000000 & 5.051758 & -1.207415 & & & & \\
\hline C & 0.000000 & 3.655735 & -1.207224 & & & & \\
\hline C & 0.000000 & -3.655735 & 1.207224 & & & & \\
\hline C & 0.000000 & -5.051758 & 1.207415 & & & & \\
\hline C & 0.000000 & -5.753410 & 0.000000 & & & & \\
\hline C & 0.000000 & -5.051758 & -1.207415 & & & & \\
\hline C & 0.000000 & -3.655735 & -1.207224 & & & & \\
\hline
\end{tabular}

5 (complex, with constraint, RB3LYP/6-31G*, $C_{2 h}$ )

\begin{tabular}{|l|rrr|l|rrr|}
\hline & $\mathrm{x}$ & $\mathrm{y}$ & $\mathrm{z}$ & & $\mathrm{x}$ & $\mathrm{y}$ & $\mathrm{z}$ \\
\hline $\mathrm{C}$ & -2.400585 & 7.166988 & 0.000000 & $\mathrm{C}$ & 1.498789 & 5.535816 & 2.450630 \\
$\mathrm{C}$ & -2.156724 & 6.531923 & 1.220346 & $\mathrm{C}$ & -1.512652 & -5.522172 & 0.000000 \\
$\mathrm{C}$ & -1.702262 & 5.192856 & 1.252477 & $\mathrm{C}$ & -1.746365 & -4.845487 & -1.237420 \\
$\mathrm{C}$ & -1.440934 & 4.441005 & 2.437080 & $\mathrm{C}$ & -1.498789 & -5.535816 & -2.450630 \\
$\mathrm{C}$ & -1.014832 & 3.111510 & 2.409289 & $\mathrm{C}$ & -1.071972 & -6.860641 & -2.443768 \\
$\mathrm{C}$ & -0.774233 & 2.482074 & 1.175479 & $\mathrm{C}$ & -0.821359 & -7.517472 & -1.242291
\end{tabular}




\begin{tabular}{|c|c|c|c|c|c|c|c|}
\hline C & -1.023159 & 3.230148 & 0.000000 & C & -1.052884 & -6.875077 & 0.000000 \\
\hline C & -0.774233 & 2.482074 & -1.175479 & C & -0.821359 & -7.517472 & 1.242291 \\
\hline C & -1.014832 & 3.111510 & -2.409289 & C & -1.071972 & -6.860641 & 2.443768 \\
\hline C & -1.440934 & 4.441005 & -2.437080 & C & -1.498789 & -5.535816 & 2.450630 \\
\hline C & -1.702262 & 5.192856 & -1.252477 & C & -1.746365 & -4.845487 & 1.237420 \\
\hline C & -2.156724 & 6.531923 & -1.220346 & C & -2.192467 & -3.501099 & 1.207836 \\
\hline C & -1.470513 & 4.551865 & 0.000000 & C & -2.442009 & -2.856940 & 0.000000 \\
\hline C & -0.365628 & 1.144995 & 0.721199 & C & -2.192467 & -3.501099 & -1.207836 \\
\hline C & -0.365628 & 1.144995 & -0.721199 & $\mathrm{H}$ & -1.624184 & 4.914525 & 3.398494 \\
\hline C & 0.000000 & 0.000000 & -1.446799 & $\mathrm{H}$ & -1.624184 & 4.914525 & -3.398494 \\
\hline C & 0.365628 & -1.144995 & -0.721199 & $\mathrm{H}$ & 1.624184 & -4.914525 & 3.398494 \\
\hline C & 0.365628 & -1.144995 & 0.721199 & $\mathrm{H}$ & 1.624184 & -4.914525 & -3.398494 \\
\hline C & 0.000000 & 0.000000 & 1.446799 & $\mathrm{H}$ & -2.768717 & 8.190068 & 0.000000 \\
\hline C & 1.023159 & -3.230148 & 0.000000 & $\mathrm{H}$ & -2.357042 & 7.052790 & 2.153256 \\
\hline C & 0.774233 & -2.482074 & 1.175479 & $\mathrm{H}$ & -2.357042 & 7.052790 & -2.153256 \\
\hline C & 1.014832 & -3.111510 & 2.409289 & $\mathrm{H}$ & -0.858884 & 2.588745 & 3.346638 \\
\hline C & 1.440934 & -4.441005 & 2.437080 & $\mathrm{H}$ & -0.858884 & 2.588745 & -3.346638 \\
\hline C & 1.702262 & -5.192856 & 1.252477 & $\mathrm{H}$ & 0.858884 & -2.588745 & 3.346638 \\
\hline C & 2.156724 & -6.531923 & 1.220346 & $\mathrm{H}$ & 2.357042 & -7.052790 & 2.153256 \\
\hline C & 2.400585 & -7.166988 & 0.000000 & $\mathrm{H}$ & 2.768717 & -8.190068 & 0.000000 \\
\hline C & 2.156724 & -6.531923 & -1.220346 & $\mathrm{H}$ & 2.357042 & -7.052790 & -2.153256 \\
\hline C & 1.702262 & -5.192856 & -1.252477 & $\mathrm{H}$ & 0.858884 & -2.588745 & -3.346638 \\
\hline C & 1.440934 & -4.441005 & -2.437080 & $\mathrm{H}$ & 2.117904 & 0.346899 & 3.113288 \\
\hline C & 1.014832 & -3.111510 & -2.409289 & $\mathrm{H}$ & 2.121308 & 0.357011 & 5.593451 \\
\hline C & 0.774233 & -2.482074 & -1.175479 & $\mathrm{H}$ & 0.000000 & 0.000000 & 6.843848 \\
\hline C & 1.470513 & -4.551865 & 0.000000 & $\mathrm{H}$ & -2.121308 & -0.357011 & 5.593451 \\
\hline C & 0.000000 & 0.000000 & 2.941937 & $\mathrm{H}$ & -2.117904 & -0.346899 & 3.113288 \\
\hline C & 0.000000 & 0.000000 & -2.941937 & $\mathrm{H}$ & 2.117904 & 0.346899 & -3.113288 \\
\hline C & 1.189305 & 0.199916 & 3.658575 & $\mathrm{H}$ & 2.121308 & 0.357011 & -5.593451 \\
\hline C & 1.190220 & 0.200759 & 5.054669 & $\mathrm{H}$ & 0.000000 & 0.000000 & -6.843848 \\
\hline C & 0.000000 & 0.000000 & 5.757066 & $\mathrm{H}$ & -2.121308 & -0.357011 & -5.593451 \\
\hline C & -1.190220 & -0.200759 & 5.054669 & $\mathrm{H}$ & -2.117904 & -0.346899 & -3.113288 \\
\hline C & -1.189305 & -0.199916 & 3.658575 & $\mathrm{H}$ & 2.368043 & 2.986217 & 2.148309 \\
\hline C & 1.189305 & 0.199916 & -3.658575 & $\mathrm{H}$ & 2.791767 & 1.828998 & 0.000000 \\
\hline C & 1.190220 & 0.200759 & -5.054669 & $\mathrm{H}$ & 2.368043 & 2.986217 & -2.148309 \\
\hline C & 0.000000 & 0.000000 & -5.757066 & $\mathrm{H}$ & 1.685332 & 5.025544 & -3.391507 \\
\hline C & -1.190220 & -0.200759 & -5.054669 & $\mathrm{H}$ & 0.904296 & 7.377504 & -3.385070 \\
\hline C & -1.189305 & -0.199916 & -3.658575 & $\mathrm{H}$ & 0.481480 & 8.549571 & -1.243565 \\
\hline C & 1.512652 & 5.522172 & 0.000000 & $\mathrm{H}$ & 0.481480 & 8.549571 & 1.243565 \\
\hline C & 1.746365 & 4.845487 & 1.237420 & $\mathrm{H}$ & 0.904296 & 7.377504 & 3.385070 \\
\hline C & 2.192467 & 3.501099 & 1.207836 & $\mathrm{H}$ & 1.685332 & 5.025544 & 3.391507 \\
\hline C & 2.442009 & 2.856940 & 0.000000 & $\mathrm{H}$ & -1.685332 & -5.025544 & -3.391507 \\
\hline C & 2.192467 & 3.501099 & -1.207836 & $\mathrm{H}$ & -0.904296 & -7.377504 & -3.385070 \\
\hline C & 1.746365 & 4.845487 & -1.237420 & $\mathrm{H}$ & -0.481480 & -8.549571 & -1.243565 \\
\hline C & 1.498789 & 5.535816 & -2.450630 & $\mathrm{H}$ & -0.481480 & -8.549571 & 1.243565 \\
\hline C & 1.071972 & 6.860641 & -2.443768 & $\mathrm{H}$ & -0.904296 & -7.377504 & 3.385070 \\
\hline C & 0.821359 & 7.517472 & -1.242291 & $\mathrm{H}$ & -1.685332 & -5.025544 & 3.391507 \\
\hline C & 1.052884 & 6.875077 & 0.000000 & $\mathrm{H}$ & -2.368043 & -2.986217 & 2.148309 \\
\hline C & 0.821359 & 7.517472 & 1.242291 & $\mathrm{H}$ & -2.791767 & -1.828998 & 0.000000 \\
\hline C & 1.071972 & 6.860641 & 2.443768 & $\mathrm{H}$ & -2.368043 & -2.986217 & -2.148309 \\
\hline
\end{tabular}


5 (complex, without constraint, RB3LYP/6-31G*, $C_{2 h}$ )

\begin{tabular}{|c|c|c|c|c|c|c|c|}
\hline & $x$ & $y$ & $z$ & & $x$ & $y$ & $z$ \\
\hline C & -2.511549 & 7.128669 & 0.000000 & C & 1.559417 & 5.593268 & 2.451244 \\
\hline C & -2.271225 & 6.492633 & 1.220814 & C & -1.592129 & -5.586186 & 0.000000 \\
\hline C & -1.809348 & 5.156203 & 1.252785 & C & -1.827149 & -4.910124 & -1.237736 \\
\hline C & -1.530344 & 4.410182 & 2.437635 & C & -1.559417 & -5.593268 & -2.451244 \\
\hline C & -1.078419 & 3.089766 & 2.409794 & C & -1.096163 & -6.905622 & -2.443746 \\
\hline C & -0.847468 & 2.456265 & 1.175356 & C & -0.844557 & -7.562163 & -1.242114 \\
\hline C & -1.116646 & 3.198322 & 0.000000 & C & -1.093656 & -6.925867 & 0.000000 \\
\hline C & -0.847468 & 2.456265 & -1.175356 & C & -0.844557 & -7.562163 & 1.242114 \\
\hline C & -1.078419 & 3.089766 & -2.409794 & C & -1.096163 & -6.905622 & 2.443746 \\
\hline C & -1.530344 & 4.410182 & -2.437635 & $\mathrm{C}$ & -1.559417 & -5.593268 & 2.451244 \\
\hline C & -1.809348 & 5.156203 & -1.252785 & $C$ & -1.827149 & -4.910124 & 1.237736 \\
\hline C & -2.271225 & 6.492633 & -1.220814 & $C$ & -2.295527 & -3.573258 & 1.208514 \\
\hline C & -1.587582 & 4.511783 & 0.000000 & C & -2.541342 & -2.928352 & 0.000000 \\
\hline C & -0.399610 & 1.133976 & 0.721900 & $\mathrm{C}$ & -2.295527 & -3.573258 & -1.208514 \\
\hline C & -0.399610 & 1.133976 & -0.721900 & $\mathrm{H}$ & -1.704657 & 4.886574 & 3.399393 \\
\hline C & 0.000000 & 0.000000 & -1.447038 & $\mathrm{H}$ & -1.704657 & 4.886574 & -3.399393 \\
\hline C & 0.399610 & -1.133976 & -0.721900 & $\mathrm{H}$ & 1.704657 & -4.886574 & 3.399393 \\
\hline C & 0.399610 & -1.133976 & 0.721900 & $\mathrm{H}$ & 1.704657 & -4.886574 & -3.399393 \\
\hline C & 0.000000 & 0.000000 & 1.447038 & $\mathrm{H}$ & -2.879822 & 8.151680 & 0.000000 \\
\hline C & 1.116646 & -3.198322 & 0.000000 & $\mathrm{H}$ & -2.465684 & 7.015992 & 2.153672 \\
\hline C & 0.847468 & -2.456265 & 1.175356 & $\mathrm{H}$ & -2.465684 & 7.015992 & -2.153672 \\
\hline C & 1.078419 & -3.089766 & 2.409794 & $\mathrm{H}$ & -0.899064 & 2.574636 & 3.347181 \\
\hline C & 1.530344 & -4.410182 & 2.437635 & $\mathrm{H}$ & -0.899064 & 2.574636 & -3.347181 \\
\hline C & 1.809348 & -5.156203 & 1.252785 & $\mathrm{H}$ & 0.899064 & -2.574636 & 3.347181 \\
\hline C & 2.271225 & -6.492633 & 1.220814 & $\mathrm{H}$ & 2.465684 & -7.015992 & 2.153672 \\
\hline C & 2.511549 & -7.128669 & 0.000000 & $\mathrm{H}$ & 2.879822 & -8.151680 & 0.000000 \\
\hline C & 2.271225 & -6.492633 & -1.220814 & $\mathrm{H}$ & 2.465684 & -7.015992 & -2.153672 \\
\hline C & 1.809348 & -5.156203 & -1.252785 & $\mathrm{H}$ & 0.899064 & -2.574636 & -3.347181 \\
\hline C & 1.530344 & -4.410182 & -2.437635 & $\mathrm{H}$ & 2.109500 & 0.393702 & 3.112889 \\
\hline C & 1.078419 & -3.089766 & -2.409794 & $\mathrm{H}$ & 2.113012 & 0.403183 & 5.593372 \\
\hline C & 0.847468 & -2.456265 & -1.175356 & $\mathrm{H}$ & 0.000000 & 0.000000 & 6.843705 \\
\hline C & 1.587582 & -4.511783 & 0.000000 & $\mathrm{H}$ & -2.113012 & -0.403183 & 5.593372 \\
\hline C & 0.000000 & 0.000000 & 2.942013 & $\mathrm{H}$ & -2.109500 & -0.393702 & 3.112889 \\
\hline C & 0.000000 & 0.000000 & -2.942013 & $\mathrm{H}$ & 2.109500 & 0.393702 & -3.112889 \\
\hline C & 1.184700 & 0.225794 & 3.658479 & $\mathrm{H}$ & 2.113012 & 0.403183 & -5.593372 \\
\hline C & 1.185589 & 0.226619 & 5.054523 & $\mathrm{H}$ & 0.000000 & 0.000000 & -6.843705 \\
\hline C & 0.000000 & 0.000000 & 5.756918 & $\mathrm{H}$ & -2.113012 & -0.403183 & -5.593372 \\
\hline C & -1.185589 & -0.226619 & 5.054523 & $\mathrm{H}$ & -2.109500 & -0.393702 & -3.112889 \\
\hline C & -1.184700 & -0.225794 & 3.658479 & $\mathrm{H}$ & 2.470659 & 3.057837 & 2.148907 \\
\hline C & 1.184700 & 0.225794 & -3.658479 & $\mathrm{H}$ & 2.897119 & 1.902285 & 0.000000 \\
\hline C & 1.185589 & 0.226619 & -5.054523 & $\mathrm{H}$ & 2.470659 & 3.057837 & -2.148907 \\
\hline C & 0.000000 & 0.000000 & -5.756918 & $\mathrm{H}$ & 1.745224 & 5.083196 & -3.392453 \\
\hline C & -1.185589 & -0.226619 & -5.054523 & $\mathrm{H}$ & 0.907792 & 7.415424 & -3.384963 \\
\hline C & -1.184700 & -0.225794 & -3.658479 & $\mathrm{H}$ & 0.475909 & 8.584358 & -1.243711 \\
\hline C & 1.592129 & 5.586186 & 0.000000 & $\mathrm{H}$ & 0.475909 & 8.584358 & 1.243711 \\
\hline C & 1.827149 & 4.910124 & 1.237736 & $\mathrm{H}$ & 0.907792 & 7.415424 & 3.384963 \\
\hline C & 2.295527 & 3.573258 & 1.208514 & $\mathrm{H}$ & 1.745224 & 5.083196 & 3.392453 \\
\hline C & 2.541342 & 2.928352 & 0.000000 & $\mathrm{H}$ & -1.745224 & -5.083196 & -3.392453 \\
\hline
\end{tabular}




\begin{tabular}{|l|rrr|l|lll|}
$\mathrm{C}$ & 2.295527 & 3.573258 & -1.208514 & $\mathrm{H}$ & -0.907792 & -7.415424 & -3.384963 \\
$\mathrm{C}$ & 1.827149 & 4.910124 & -1.237736 & $\mathrm{H}$ & -0.475909 & -8.584358 & -1.243711 \\
$\mathrm{C}$ & 1.559417 & 5.593268 & -2.451244 & $\mathrm{H}$ & -0.475909 & -8.584358 & 1.243711 \\
$\mathrm{C}$ & 1.096163 & 6.905622 & -2.443746 & $\mathrm{H}$ & -0.907792 & -7.415424 & 3.384963 \\
$\mathrm{C}$ & 0.844557 & 7.562163 & -1.242114 & $\mathrm{H}$ & -1.745224 & -5.083196 & 3.392453 \\
$\mathrm{C}$ & 1.093656 & 6.925867 & 0.000000 & $\mathrm{H}$ & -2.470659 & -3.057837 & 2.148907 \\
$\mathrm{C}$ & 0.844557 & 7.562163 & 1.242114 & $\mathrm{H}$ & -2.897119 & -1.902285 & 0.000000 \\
$\mathrm{C}$ & 1.096163 & 6.905622 & 2.443746 & $\mathrm{H}$ & -2.470659 & -3.057837 & -2.148907 \\
\hline
\end{tabular}

\begin{tabular}{|c|c|c|c|c|c|c|c|}
\hline \multicolumn{8}{|c|}{ BPBR without substitution (RB3LYP/6-31G*, $D_{2 h}$ ) } \\
\hline & $x$ & $\mathrm{y}$ & z & & $x$ & $\mathrm{y}$ & z \\
\hline $\mathrm{C}$ & 0.000000 & 0.000000 & 7.530911 & $\mathrm{H}$ & 0.000000 & 3.404970 & 5.149545 \\
\hline C & 0.000000 & 1.221959 & 6.850537 & $\mathrm{H}$ & 0.000000 & -3.404970 & 5.149545 \\
\hline C & 0.000000 & 1.253421 & 5.441962 & $\mathrm{H}$ & 0.000000 & 3.404970 & -5.149545 \\
\hline C & 0.000000 & 2.444560 & 4.638955 & $\mathrm{H}$ & 0.000000 & -3.404970 & -5.149545 \\
\hline C & 0.000000 & 2.417129 & 3.252476 & $\mathrm{H}$ & 0.000000 & 0.000000 & 8.617672 \\
\hline C & 0.000000 & 1.173444 & 2.577278 & $\mathrm{H}$ & 0.000000 & 2.153534 & 7.411842 \\
\hline C & 0.000000 & 0.000000 & 3.371082 & $\mathrm{H}$ & 0.000000 & -2.153534 & 7.411842 \\
\hline C & 0.000000 & -1.173444 & 2.577278 & $\mathrm{H}$ & 0.000000 & 3.353575 & 2.699958 \\
\hline C & 0.000000 & -2.417129 & 3.252476 & $\mathrm{H}$ & 0.000000 & -3.353575 & 2.699958 \\
\hline C & 0.000000 & -2.444560 & 4.638955 & $\mathrm{H}$ & 0.000000 & 2.524815 & 0.000000 \\
\hline C & 0.000000 & -1.253421 & 5.441962 & $\mathrm{H}$ & 0.000000 & -2.524815 & 0.000000 \\
\hline C & 0.000000 & -1.221959 & 6.850537 & $\mathrm{H}$ & 0.000000 & 3.353575 & -2.699958 \\
\hline C & 0.000000 & 0.000000 & 4.763781 & $\mathrm{H}$ & 0.000000 & 2.153534 & -7.411842 \\
\hline C & 0.000000 & 0.726458 & 1.202419 & $\mathrm{H}$ & 0.000000 & 0.000000 & -8.617672 \\
\hline C & 0.000000 & -0.726458 & 1.202419 & $\mathrm{H}$ & 0.000000 & -2.153534 & -7.411842 \\
\hline C & 0.000000 & -1.436469 & 0.000000 & $\mathrm{H}$ & 0.000000 & -3.353575 & -2.699958 \\
\hline C & 0.000000 & -0.726458 & -1.202419 & & & & \\
\hline C & 0.000000 & 0.726458 & -1.202419 & & & & \\
\hline C & 0.000000 & 1.436469 & 0.000000 & & & & \\
\hline C & 0.000000 & 0.000000 & -3.371082 & & & & \\
\hline C & 0.000000 & 1.173444 & -2.577278 & & & & \\
\hline C & 0.000000 & 2.417129 & -3.252476 & & & & \\
\hline C & 0.000000 & 2.444560 & -4.638955 & & & & \\
\hline C & 0.000000 & 1.253421 & -5.441962 & & & & \\
\hline C & 0.000000 & 1.221959 & -6.850537 & & & & \\
\hline C & 0.000000 & 0.000000 & -7.530911 & & & & \\
\hline C & 0.000000 & -1.221959 & -6.850537 & & & & \\
\hline C & 0.000000 & -1.253421 & -5.441962 & & & & \\
\hline C & 0.000000 & -2.444560 & -4.638955 & & & & \\
\hline C & 0.000000 & -2.417129 & -3.252476 & & & & \\
\hline C & 0.000000 & -1.173444 & -2.577278 & & & & \\
\hline C & 0.000000 & 0.000000 & -4.763781 & & & & \\
\hline
\end{tabular}


BPBR without substitution (RB3LYP/6-311+G(2d), $D_{2 h}$ )

\begin{tabular}{|c|c|c|c|c|c|c|c|}
\hline & $x$ & $\mathrm{y}$ & $z$ & & $x$ & $\mathrm{y}$ & z \\
\hline C & 0.000000 & 0.000000 & 7.508480 & $\mathrm{H}$ & 0.000000 & 3.395786 & 5.134591 \\
\hline C & 0.000000 & 1.217572 & 6.831051 & $\mathrm{H}$ & 0.000000 & -3.395786 & 5.134591 \\
\hline C & 0.000000 & 1.249038 & 5.427087 & $\mathrm{H}$ & 0.000000 & 3.395786 & -5.134591 \\
\hline C & 0.000000 & 2.437158 & 4.626083 & $\mathrm{H}$ & 0.000000 & -3.395786 & -5.134591 \\
\hline C & 0.000000 & 2.409859 & 3.245148 & $\mathrm{H}$ & 0.000000 & 0.000000 & 8.592505 \\
\hline C & 0.000000 & 1.170161 & 2.570970 & $\mathrm{H}$ & 0.000000 & 2.146096 & 7.392338 \\
\hline C & 0.000000 & 0.000000 & 3.362153 & $\mathrm{H}$ & 0.000000 & -2.146096 & 7.392338 \\
\hline C & 0.000000 & -1.170161 & 2.570970 & $\mathrm{H}$ & 0.000000 & 3.344914 & 2.695304 \\
\hline C & 0.000000 & -2.409859 & 3.245148 & $\mathrm{H}$ & 0.000000 & -3.344914 & 2.695304 \\
\hline C & 0.000000 & -2.437158 & 4.626083 & $\mathrm{H}$ & 0.000000 & 2.518082 & 0.000000 \\
\hline C & 0.000000 & -1.249038 & 5.427087 & $\mathrm{H}$ & 0.000000 & -2.518082 & 0.000000 \\
\hline C & 0.000000 & -1.217572 & 6.831051 & $\mathrm{H}$ & 0.000000 & 3.344914 & -2.695304 \\
\hline C & 0.000000 & 0.000000 & 4.750397 & $\mathrm{H}$ & 0.000000 & 2.146096 & -7.392338 \\
\hline C & 0.000000 & 0.724767 & 1.198485 & $\mathrm{H}$ & 0.000000 & 0.000000 & -8.592505 \\
\hline C & 0.000000 & -0.724767 & 1.198485 & $\mathrm{H}$ & 0.000000 & -2.146096 & -7.392338 \\
\hline C & 0.000000 & -1.432087 & 0.000000 & $\mathrm{H}$ & 0.000000 & -3.344914 & -2.695304 \\
\hline C & 0.000000 & -0.724767 & -1.198485 & & & & \\
\hline C & 0.000000 & 0.724767 & -1.198485 & & & & \\
\hline C & 0.000000 & 1.432087 & 0.000000 & & & & \\
\hline C & 0.000000 & 0.000000 & -3.362153 & & & & \\
\hline C & 0.000000 & 1.170161 & -2.570970 & & & & \\
\hline C & 0.000000 & 2.409859 & -3.245148 & & & & \\
\hline C & 0.000000 & 2.437158 & -4.626083 & & & & \\
\hline C & 0.000000 & 1.249038 & -5.427087 & & & & \\
\hline C & 0.000000 & 1.217572 & -6.831051 & & & & \\
\hline C & 0.000000 & 0.000000 & -7.508480 & & & & \\
\hline C & 0.000000 & -1.217572 & -6.831051 & & & & \\
\hline C & 0.000000 & -1.249038 & -5.427087 & & & & \\
\hline C & 0.000000 & -2.437158 & -4.626083 & & & & \\
\hline C & 0.000000 & -2.409859 & -3.245148 & & & & \\
\hline C & 0.000000 & -1.170161 & -2.570970 & & & & \\
\hline C & 0.000000 & 0.000000 & -4.750397 & & & & \\
\hline
\end{tabular}


Total energies of all DFT-calculated structures

\begin{tabular}{|c|c|c|}
\hline Structures & $\left\langle S^{2}\right\rangle$ & \begin{tabular}{|l} 
Total Energies (hartree) \\
\end{tabular} \\
\hline \multirow[t]{2}{*}{2 (RB3LYP/6-31G*, $\left.D_{2 h}\right)$} & 0 & -1691.20156 \\
\hline & 2.075 & -1691.19323 \\
\hline \multirow{2}{*}{2 (UB3LYP/6-31G*, $\left.D_{2 h}\right)$} & 0.638 & -1691.20377 \\
\hline & 2.077 & -1691.19485 \\
\hline \multirow[t]{2}{*}{2 X-ray structure (UB3LYP/6-31G*) } & 0.707 & -1691.13883 \\
\hline & 2.076 & -1691.13134 \\
\hline 2 (1-D chain, RB3LYP/6-31G*) & 0 & -1691.19166 \\
\hline 2 (monocation, UB3LYP/6-31G*, $D_{2 h}$ ) & 0.784 & -1691.00348 \\
\hline 2 (dication, RB3LYP/6-31G*, $D_{2 h}$ ) & 0 & -1690.68994 \\
\hline 2 (dianion, RB3LYP/6-31G*, $D_{2 h}$ ) & 0 & -1691.23506 \\
\hline \multirow{2}{*}{3 (RB3LYP/6-31G*, $\left.D_{2 h}\right)$} & 0 & -1858.07558 \\
\hline & 2.072 & -1858.06764 \\
\hline \multirow[t]{2}{*}{3 (UB3LYP/6-31G*, $D_{2 h}$ ) } & 0.617 & -1858.07760 \\
\hline & 2.074 & -1858.06850 \\
\hline 4 (RB3LYP/6-31G*, $\left.D_{2 h}\right)$ & 0 & -1461.35698 \\
\hline 5 (complex, with constraint, RB3LYP/6-31G*, $C_{2 h}$ ) & 0 & -2692.80922 \\
\hline 5 (complex, with constraint, UB3LYP/6-31G*, $C_{2 h}$ ) & 6.182 & -2692.76312 \\
\hline $\begin{array}{l}5 \text { (complex, without constraint, RB3L YP/6-31G*, } \\
C_{2 h} \text { ) }\end{array}$ & 0 & -2692.81239 \\
\hline BPBR without substitution (RB3LYP/6-31G*, $D_{2 h}$ ) & 0 & -1229.09627 \\
\hline $\begin{array}{l}\text { BPBR without substitution (RB3LYP/6- } \\
\left.311+\mathrm{G}(2 \mathrm{~d}), D_{2 h}\right)\end{array}$ & 0 & -1229.37711 \\
\hline
\end{tabular}




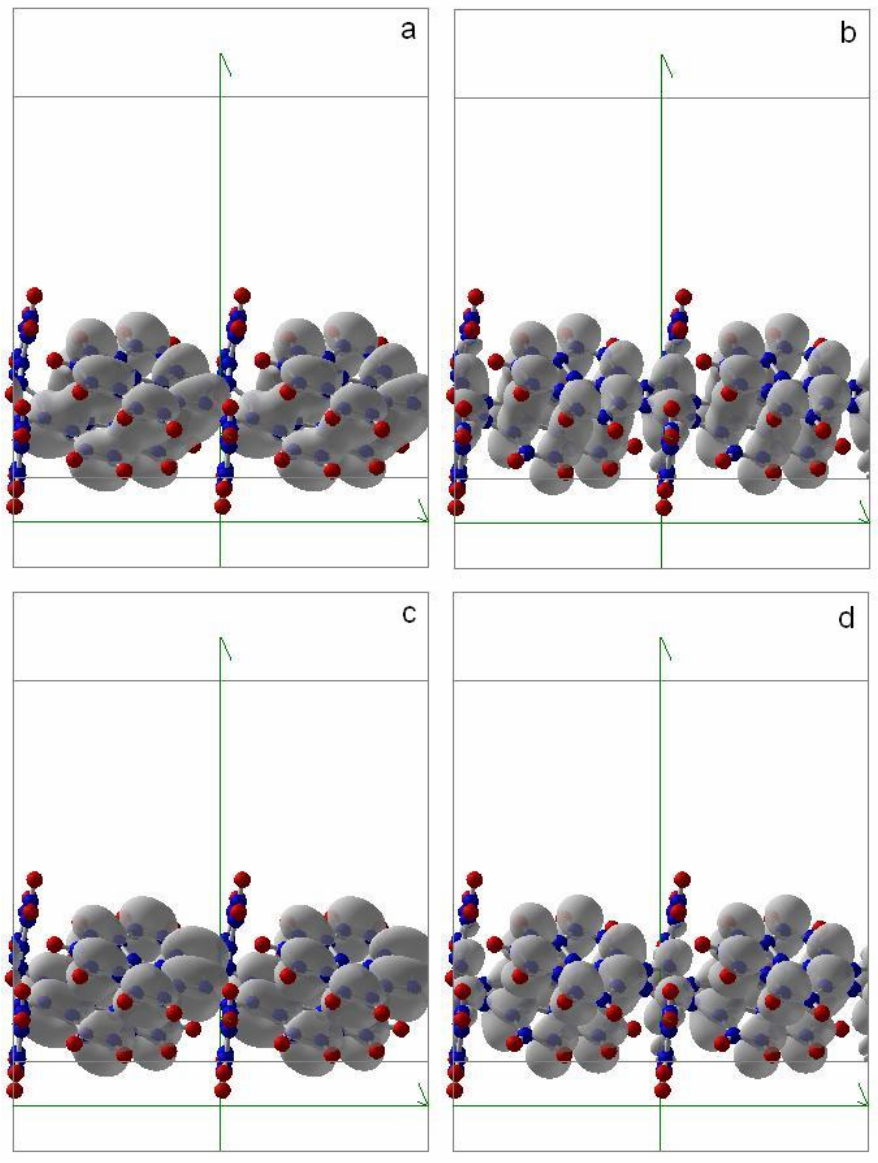

Electron density isosurfaces of the Kohn-Sham crystal orbitals at the zone edges for the two bands closest to the Fermi level calculated with VASP program. The crystal orbitals a, b, c, and d are in the order of increasing energy: a $(k=0), \mathrm{b}(k=\pi / \mathbf{b} \mid), \mathrm{c}(k=$ $\pi\langle\mathbf{b}|), \mathrm{d}(k=0)$. The crystal orbitals a and c are derived from HOMO of BPBR, while b and $d$ are derived from LUMO of BPBR (see Figure 2). Note the in-phase $\pi-\pi$ overlap of a and b, and the out-of-phase $\pi-\pi$ overlap of c and d. Similar to the analysis in the Supporting Information of ref 12a, this phase relationship indicates that the intramolecular transfer integral is smaller than the intermolecular one. 